$\mathrm{Zu}$ den beiden ersten Argumenten wurde bereits oben Stellung genommen: Sie verkennen erstens, dass sich nicht einigungsbereite Fraktionen letztlich auch durch Verfassungsbestimmungen nicht zur Kreation beziehungsweise Unterstützung einer stabilen Regierung zwingen lassen. Zweitens geht die Vorstellung von politisch „unabhängigen“ Verfassungsinstanzen von einem rein formalen Verfassungsverständnis aus, das die enge Verknüpfung von Bundestagsmehrheit und „ihrem“ Kanzler außer Acht lässt. Drittens kann der Verweis auf die Situation in den genannten Ländern am wenigsten überzeugen: Die häufigen Regierungswechsel in Italien bis zu Beginn der 1990er Jahre etwa waren keineswegs auf vorzeitige Beendigungen der Wahlperioden zurückzuführen, sondern auf eine davon völlig unabhängige Instabilität des italienischen Parteiensystems.

In der Frage der notwendigen Mehrheit, die einer Selbstauflösung zustimmen muss, spricht gerade die auch von Pieper angeführte ansonsten drohende Majorisierung kleinerer Parteien für ein im Vergleich zu sonstigen Parlamentsbeschlüssen erhöhtes Quorum (zwei Drittel). Bemerkenswert ist, dass in fast allen Bundesländern, in denen es sowohl ein Selbstaufösungsrecht mit qualifizierter Mehrheit als auch die Möglichkeit der mit einfacher Mehrheit abzulehnenden Vertrauensfrage gibt, letztere bislang nie genutzt wurde, um eine Parlamentsauflösung durch die Mehrheitsfraktionen gegen den Willen der größeren Oppositionsfraktion durchzusetzen. ${ }^{29}$ Hier hat sich also die Selbstauflösung als ein eher konsensual genutztes Mittel gezeigt. In jedem Fall würde es den bisher notwendigen, für die Bürger kaum nachvollziehbaren Umweg über die Vertrauensfrage ersparen, wenn ohnehin die große Mehrheit aller Parlamentsparteien der Meinung ist, Neuwahlen anberaumen zu müssen.

29 Vgl. Sven Leunig, a.a.O., S. 127 - 140.

\title{
Ein Blick über die Grenzen hilft manchmal weiter. Eine Replik auf Gerd Strohmeier in Heft 3/2007 der ZParl ${ }^{*}$
}

\author{
Stefan Köppl
}

Gerd Strohmeiers Vorschlag zu einer Reform des deutschen Wahlrechts hat bereits massive Kritik geerntet. Frank Decker zeigte in seiner Replik, dass Strohmeiers Problemdiagnose unvollständig beziehungsweise unzutreffend, seine Idealvorstellungen fragwürdig und seine Reformvorschläge systemwidrig sowie unrealistisch seien. ${ }^{1}$ Zudem rechnete Harald Schoen nach, dass die Vorschläge kaum dazu geeignet sind, ihre Ziele zu erreichen. ${ }^{2}$ Dem soll an

* Gerd Strohmeier, Ein Plädoyer für die ,gemäßigte Mehrheitswahl“: optimale Lösung für Deutschland, Vorbild für Österreich und andere Demokratien, in: ZParl, 38. Jg. (2007), H. 3, S. 578 $-590$.

1 Frank Decker, Konsens- oder mehrheitsdemokratischer Wandel des Parlamentarismus? Eine Replik auf Gerd Strohmeier in Heft 3/2007 der ZParl, in: ZParl, 38. Jg. (2007), H. 4, S. 857 - 861.

2 Harald Schoen, Eine optimale Lösung? Eine Replik auf Gerd Strohmeier in Heft 3/2007 der ZParl, in: ZParl, 38. Jg. (2007), H. 4, S. $862-865$. 
dieser Stelle hinzugefügt werden, dass der Beitrag den Stand der Forschung ignoriert und seine Gerechtigkeitsargumentation mit Inkonsistenzen zu kämpfen hat. So lässt sich etwa anhand der italienischen Parlamentswahlen zwischen 1994 und 2001 zeigen, welche Strategien die politischen Akteure entwickeln könnten, um die von Strohmeier implizierten Wirkungen zu unterlaufen.

Für seinen Vorschlag gibt es bereits einen Testfall in der Realität: Die „gemäßigte Mehrheitswahl“ entspricht (zumindest in der „schwächeren“ Variante, in der nur drei Viertel statt fünf Sechstel der Abgeordneten nach Mehrheitswahl gewählt werden) im Wesentlichen dem Wahlrecht, das zwischen 1993 und 2005 in Italien bei der Wahl zur Abgeordnetenkammer galt ${ }^{3}$ : Hier wurden drei Viertel der Abgeordneten in Einerwahlkreisen nach relativer Mehrheitswahl gewählt, ein Viertel über Parteilisten nach Verhältniswahl. ${ }^{4}$

Dieses Wahlrecht wurde in Italien aus genau denselben Gründen beschlossen, die Strohmeier für seinen Reformvorschlag anführt: Statt einer großen kartellartigen Dauerkoalition der Mitte (im Unterschied zu Deutschland nicht aus drei, sondern aus fünf Parteien) sollte es zu Regierungswechseln kommen und idealerweise eine Partei die Regierung stellen; so würden sowohl Koalitionsgerangel als auch Koalitionsbildungen unabhängig vom Wählervotum (in Strohmeiers Diktion: „Unregierbarkeit“ und „Wahlsiegparadoxon“) der Vergangenheit angehören. Schließlich sollte die Verhältniskomponente dennoch die Existenz kleinerer Parteien sichern.

Es ist befremdlich, dass Strohmeier trotz dieser Parallelen den Testfall Italien mit keinem Wort erwähnt, obwohl sein Vorschlag dort in drei nationalen Parlamentswahlen (1994, 1996 und 2001) erprobt wurde - vielleicht weil es seine abstrakten Überlegungen mit einer Wirklichkeit konfrontiert, die zumindest im italienischen Fall das genaue Gegenteil von dem erzeugte, was er für Deutschland als Effekt voraussagt:

(1) Seit der Reform gab es nach jeder Wahl in Italien einen Regierungswechsel. Dieses Ziel wurde erreicht. Allerdings kamen die Wechsel nicht durch Wählerwanderungen zustande, sondern durch Taktik, denn entscheidend für den Gewinn der Einerwahlkreise war es, ein möglichst breites Bündnis zu schmieden. Schon das Ausscheren einer kleineren Partei aus einer Allianz konnte wahlentscheidend sein, wie es 1996 und 2001 der Fall war.

(2) Die Regierungen wurden folglich nicht von einer Partei gebildet, sondern von Koalitionen, die zum Teil noch mehr Parteien umfassten als vor der Wahlrechtsreform. Wegen der rein quantitativ orientierten taktischen Bündnisbildung stellten sich die Regierungskoalitionen inhaltlich sogar noch heterogener dar als zuvor, so dass von einer Verbesserung der Regierbarkeit kaum gesprochen werden kann.

3 Vgl. zum Folgenden exemplarisch Stefano Bartolini / Alessandro Chiaramonte / Roberto D'Alimonte, The Italian Party System between Parties and Coalitions, in: West European Politics, 27. Jg. (2004), H. 1, S. 1 - 19 und Roberto D'Alimonte / Stefano Bartolini (Hrsg.), Maggioritario finalmente? La transizione elettorale 1994 - 2001, Bologna 2002.

4 Im Unterschied zu Strohmeiers Vorschlag gab es im italienischen Fall eine geringfügige Verrechnung der Stimmen zwischen den beiden Teilen (die die Chancen kleiner Parteien bei der Verhältniskomponente erhöhte) und eine Sperrklausel bei der Verhältniswahl. Diese beiden Unterschiede sind allerdings für die vorliegende Betrachtung von geringer Bedeutung, weil (a) nur eine Verrechnung des Stimmenvorsprungs stattfand (also nicht auf der Mandatsebene und daher ganz anders als in der personalisierten Verhältniswahl), (b) in zahlreichen Fällen diese Verrechnung durch Tarnlisten (so genannte liste civetta, Eulenlisten) umgangen wurde und (c) die Effekte des Wahlsystems in erster Linie durch andere Mechanismen, nämlich die im Folgenden geschilderten eintraten. 
(3) Die Parteienzersplitterung verstärkte sich nach der Wahlrechtsreform noch, was aber nur zum kleineren Teil auf die Verhältniskomponente zurückzuführen war. Vielmehr konnten selbst Parteien, die an der Sperrklausel bei der Listenwahl gescheitert wären, ins Parlament einziehen - nämlich gerade aufgrund der Schlüsselrolle, die ihnen die Mehrheitswahl in den Wahlkreisen zuspielte. Im Austausch für die Unterstützung eines Bündnisses handelten sie für ihre eigenen Direktkandidaten „sichere“ Wahlkreise heraus - mindestens in dem Ausmaß ihres in Umfragewerten gemessenen Stimmengewichts, meist aber überproportional darüber hinaus. Somit gab es nicht nur keinen Anreiz dafür, sich einer größeren Partei anzuschließen, sondern im Gegenteil: Die Anreizstruktur des Wahlrechts belohnte geradezu kleine selbständige Formationen. Das Ergebnis war also eine „Proportionalisierung“ der Mehrheitswahl. ${ }^{5}$ So kam zur inhaltlichen Heterogenität der Koalitionspartner auch noch eine größere Anzahl hinzu, was das Regieren zusätzlich erschwerte.

Gewiss darf man den Fall Italien als Referenzpunkt nicht überstrapazieren; zu groß sind die Unterschiede in der parteipolitischen und ideologischen Landschaft im Vergleich zu Deutschland. Aber eine kurze Auseinandersetzung mit diesem Testfall wäre durchaus sinnvoll gewesen, deckt er doch strukturelle Mechanismen auf, von denen erst einmal gezeigt werden müsste, warum sie in geringerem Ausmaße nicht auch anderswo wirken sollen: Würden nach der Einführung der gemäßigten Mehrheitswahl in Deutschland die kleineren Parteien wirklich „zur Bedeutungslosigkeit verurteilt"6. Es gibt zumindest Gründe, das zu bezweifeln.

In Italien haben die kleineren Parteien nach Einführung der Mehrheitswahl in einem Mehrparteiensystem ihr Stimmengewicht beziehungsweise ihr Erpressungspotential (die Drohung mit der Aufstellung eigener Wahlkreiskandidaten, die den Wettbewerb zwischen den größeren Parteien entscheidend beeinflussen würden) taktisch eingesetzt. Warum sollten die kleineren deutschen Parteien das nicht tun? Vice versa haben die größeren Parteien Italiens sofort erkannt, dass man sich in der Konkurrenz um die Wahlkreismandate durch taktische Bündnisse mit den kleineren Parteien Vorteile verschaffen kann. Warum sollten Union und SPD nicht auf den gleichen Gedanken kommen? Zu erwarten, dass sich die kleineren drei Parteien einfach mit ihrem Platz am Katzentisch der Verhältniswahl zufrieden geben und nicht versuchen, mit ihren Anhängern in die Entscheidung in den Wahlkreisen einzugreifen, ist naiv. Dass die bisherigen Wähler von FDP, Grünen und der Linken einfach vor dem neuen Wahlrecht kapitulieren und einen Direktkandidaten von Union oder SPD wählen, weil ihre Stimme sonst „verschenkt“ wäre, ist eine abseitige Vorstellung, die schon durch das regelmäßig zu beobachtende Stimmverhalten in der personalisierten Verhältniswahl konterkariert wird. ${ }^{7}$

Wahlentscheidend könnte dann weniger das eigene Politikangebot der großen Parteien werden als vielmehr das Geschick, sich die Unterstützung von mindestens einer der drei kleineren Parteien zu sichern, etwa indem ihnen „sichere“ Wahlkreise überlassen werden.

5 Roberto D'Alimonte, Mixed Electoral Rules, Partisan Realignment, and Party System Change in Italy, in: Matthew S. Shugart / Martin P. Wattenberg (Hrsg.), Mixed-Member Electoral Systems: The Best of Both Worlds?, Oxford 2001, S. 323 - 350.

6 Frank Decker, a.a.O., S. 859.

7 So erhielten FDP und Grüne bei der Bundestagswahl 2005 immerhin je circa fünf Prozent der Erststimmen, obwohl sie (mit Ausnahme Christian Ströbeles im Wahlkreis Berlin - Friedrichshain Kreuzberg - Prenzlauer Berg-Ost) kaum Aussichten auf den Gewinn eines Direktmandats hatten. 
Zum Beispiel könnte die SPD als Zugeständnis an die Grünen in Berlin-Kreuzberg nicht mit einem eigenen Kandidaten oder nur einem offensichtlich unterlegenen Zählkandidaten antreten. So würden auch kleinere Parteien Sitze über die Mehrheitswahl in den Wahlkreisen erhalten und die Verhältniswahl ihnen einen Anreiz geben, eigenständig zu bleiben ${ }^{8}$ und somit die Mehrheitswahl „kontaminieren“9. Sicher kann man formale Bündnisse ähnlich wie Listenverbindungen rechtlich unterbinden, aber informale Absprachen und / oder Wahlempfehlungen wohl kaum.

Dass Strohmeier den instruktiven Fall Italien ignoriert, kann noch mit Unkenntnis erklärt werden. Doch auch andere Aspekte des Forschungsstandes blendet er aus:

(1) So stellt er zwar Bezüge zu ähnlichen Wahlsystemen bis zurück zum deutschen Kaiserreich beziehungsweise zu einem nicht umgesetzten britischen Vorschlag her, übersieht aber die Ergebnisse internationaler Wahlforschung zu ähnlichen gemischten Wahlsystemen, die bereits Realität sind (über den Fall Italien hinaus). ${ }^{10}$ Deren Verfechter gehen gemeinhin von der theoretischen Annahme aus, dass die beiden Komponenten, also der Mehrheitsund der Verhältnisanteil, unabhängig voneinander gemäß ihrer Lehrbuchlogik funktionieren und durch ihre Kombination auch die ihnen zugeschriebenen Effekte gleichzeitig und damit das „Beste aus zwei Welten“ erreicht werden könnte. Diese Annahme darf aber als von der empirischen Forschung widerlegt gelten. Federico Ferrara, Erik S. Herron und Misa Nishikawa haben gezeigt, dass die Parteien umso mehr Anreize zu formellen oder informellen Absprachen haben, je größer die Mehrheitskomponente ist. ${ }^{11}$

(2) Manche Sätze lassen auf ein befremdend realitätsfernes Bild des bundesrepublikanischen Parlamentarismus schließen, so etwa: „Schließlich kann eine Partei, auch wenn sie gerecht und zahlenmäßig stark im Parlament vertreten ist, politische Entscheidungen grundsätzlich nicht beeinflussen [sic], solange sie keine Regierungspartei ist. "12 Mit den Ergebnissen der Parlamentarismusforschung der letzten Jahrzehnte ist das nicht vereinbar. In der Realität ist der schroffe Gegensatz von Regierungsmehrheit und Opposition in der Gesetzgebung eher die Ausnahme als die Regel, was auch längst Eingang in die Lehrbücher gefunden hat. ${ }^{13}$ Im Lichte seiner Überschätzung des Westminster-Modells (vgl. die Replik von Decker) scheint Strohmeier hier die Realität mit dem von den Medien, zu deren Eigenlogik notfalls auch die künstliche Konstruktion dieses Dualismus gehört, verzerrten Bild zu

8 Aber auch unabhängig von diesem Aspekt scheint eine Verschmelzung von Union und FDP beziehungsweise SPD und Grünen kaum denkbar.

9 Vgl. zur Literatur, die es zu diesem Phänomen der „contamination“ in gemischten Wahlsystemen gibt, nur zwei Beispiele: Federico Ferrara, Frogs, Mice and Mixed Electoral Institutions: Party Discipline in Italy's XIV Chamber of Deputies, in: Journal of Legislative Studies, 10. Jg. (2004), H. 4, S. 10 - 31 (auch zu den Auswirkungen der beschriebenen Effekte auf die parlamentarische Arena), und Karen E. Cox / Leonard J. Schoppa, Interaction Effects in Mixed-Member Electoral Systems: Theory and Evidence from Germany, Japan, and Italy, in: Comparative Political Studies, 35. Jg. (2002), H. 9, S. 1027 - 1053.

10 Zum Beispiel Matthew S. Shugart / Martin P. Wattenberg (Hrsg.), a.a.O., und Federico Ferrara I Erik S. Herron / Misa Nishikawa, Mixed Electoral Systems, New York 2005. Vgl. auch für den internationalen Vergleich von Wahlsystemen: Michael Gallagher / Paul Mitchell (Hrsg.), The Politics of Electoral Systems, Oxford u.a. 2005.

11 Vgl. Federico Ferrara / Erik S. Herron / Misa Nishikawa, a.a.O., S. 63.

12 Gerd Strohmeier, a.a.O., S. 583.

13 Nur ein Beispiel: Ludger Helms, Politische Opposition. Theorie und Praxis in westlichen Regierungssystemen, Opladen 2002, S. $40-69$. 
verwechseln. Politische Prozesse sind weitaus komplexer, als es das Bild der in der Schlussabstimmung unterlegenen Opposition suggeriert. So ist zum Beispiel deren wichtige Rolle bei der Thematisierung und Entwicklung von Lösungen seit einiger Zeit von der Forschung herausgearbeitet worden. ${ }^{14}$

(3) Ähnlich verhält es sich mit dem Einfluss kleiner Koalitionsparteien: „Unter diesen Umständen ist der machtpolitische Einfluss einer kleinen Regierungspartei definitiv am stärksten (letztlich sogar stärker als der der großen Regierungspartei). " 15 Hier scheint immer noch die zentrale Rolle der FDP im Zweieinhalb-Parteiensystem durch, die längst Vergangenheit ist. Der Einfluss kleiner Koalitionspartner im Regierungsprozess mag größer sein als nach den Proportionen der Sitzverteilung, beruht aber im Wesentlichen auf ihrer Vetoposition. ${ }^{16}$

(4) Analog scheint Strohmeier die innere Geschlossenheit von Union und SPD zu überschätzen, die alles andere als zentral und effektiv steuerbare „Kanzlerwahlvereine“ sind. Beide Parteien haben immer wieder mit Zerreißproben zwischen verschiedenen Flügeln zu kämpfen. Einige Nachteile, die er Koalitionsregierungen zuschreibt (zum Beispiel der Kompromisscharakter von Entscheidungen), dürften auch bei Einparteiregierungen nicht verschwinden, sondern nur in veränderter oder leicht verminderter Form weiterbestehen. Die Rolle des kleineren Koalitionspartners könnten dann die entsprechenden widerstreitenden Parteiflügel einnehmen, denen besonders bei knappen Mehrheiten auch entsprechendes Erpressungspotential zukäme, wie das Beispiel der SPD-,Abweichler“ in der Diskussion um die Agenda 2010 zeigte. ${ }^{17}$

Abschließend sei ein Blick auf Strohmeiers Gerechtigkeitsargumentation geworfen, die eine grundsätzlich problematische Sichtweise offenbart.

Erstens ist nicht einsichtig, warum der überproportionale Einfluss einer großen Partei gerechter sein soll als der überproportionale Einfluss einer kleinen Partei. Einerseits lehnt Strohmeier Koalitionsregierungen wegen des ungerecht großen Einflusses des kleinen Partners ab, andererseits scheint ihm das „Durchregieren“ einer Einparteiregierung (die von weit weniger als 50 Prozent der Wähler unterstützt wird) unproblematisch zu sein, solange es nur effizient geschieht.

Folgt man, zweitens, Strohmeiers Annahme, dass die Größenverhältnisse im deutschen Parteiensystem künftig so bleiben wie derzeit, blieben auch SPD und Union in etwa gleich

14 Martin Sebaldt, Die Thematisierungsfunktion der Opposition. Die parlamentarische Minderheit des Deutschen Bundestags als innovative Kraft im politischen System der Bundesrepublik Deutschland, Frankfurt am Main u.a. 1992. Selbst die Grünen im von der CSU dominierten Bayerischen Landtag können keinesfalls als einflusslos bezeichnet werden: Katrin Steinack, Opposition im Bayerischen Landtag 1994 - 1998. Ebenen und Strategien politischer Einflussnahme in einem hegemonialen System, Frankfurt am Main u.a. 2007. Sogar für die britische WestminsterDemokratie konstatiert dies Ludger Helms, Wettbewerb und Kooperation. Zum Verhältnis von Regierungsmehrheit und Opposition im parlamentarischen Gesetzgebungsverfahren in der Bundesrepublik Deutschland, Großbritannien und Österreich, Opladen 1997.

15 Gerd Strohmeier, a.a.O., S. 584.

16 Vgl. exemplarisch für einen ausgewogeneren Blick auf die Wirklichkeit deutscher Koalitionen die Beiträge in: Christoph Egle / Reimut Zohlnhöfer (Hrsg.), Ende des rot-grünen Projekts. Eine Bilanz der Regierung Schröder 2002 - 2005, Wiesbaden 2007.

17 Vgl. dazu exemplarisch Manfred Schwarzmeier, Umgangsstil und Regelverletzungen: Indikatoren politischer Machterosion?, in: Werner J. Patzelt / Martin Sebaldt / Uwe Kranenpohl (Hrsg.), Res publica semper reformanda. Wissenschaft und politische Bildung im Dienst des Gemeinwohls, Wiesbaden 2007, S. $291-303$. 
stark. Stellt man weiterhin die regionalen Hochburgen der großen Parteien als konstant in Rechnung, würde sich das Kopf-an-Kopf-Rennen in wenigen „Swing“-Wahlkreisen entscheiden. 2005 wechselten 25 der 299 Wahlkreise ihre parteipolitische „Farbe“. Es gäbe also ähnlich wie in den USA eine Unterscheidung zwischen Wahlkreisen, die für die Entscheidung wichtig, und solchen, die es nicht sind. Die Stimmen der Wähler in ersteren hätten - analog zu Strohmeiers Argumentation bezüglich der kleinen Koalitionsparteien ein größeres Gewicht, als ihnen „gerechterweise“ zukommen sollte.

Drittens - und wie auch schon von Schoen in seiner Replik gezeigt - gibt es keinen Grund zu erwarten, dass die von Strohmeier geforderte Reform die gewünschten Ergebnisse bringen wird. Selbst wenn es unter ihren Bedingungen möglich sein sollte, eine kleine (also Zweiparteien-)Koalition ${ }^{18}$ zu bilden, würde die Reform das genaue Gegenteil ihrer Ziele erreichen: Der angeblich ungerechte Einfluss eines kleinen Koalitionspartners bestünde weiter und würde sogar mit einer noch deutlich geringeren Mandatszahl ausgeübt. Er wäre folglich weit stärker überproportional als bisher. Es könnte sogar noch schlimmer kommen: Da in Strohmeiers Modell keine Sperrklausel vorgesehen ist, wäre sogar eine Konstellation denkbar, in der sich schwarz-gelb und rot-grün-rot fast gleich stark gegenüberstehen und die Mandate von Splitterparteien (von mindestens einem Mandat für die NPD ist auszugehen) das Zünglein an der Waage darstellen. Wie gerecht wäre das?

Diese drei Einwände sind zugespitzt, zeigen aber die grundsätzliche Problematik der Betrachtungsweise auf, wenn man den Einfluss, der Parteien aufgrund ihrer spezifischen und kontingenten Position in der Akteurskonstellation zukommt, unter Gerechtigkeitsgesichtspunkten gegen den anderer Parteien abwägt, deren Position ihnen weniger Einfluss erlaubt.

Eine Gerechtigkeitsdiskussion bezüglich des Wahlrechts ist in Deutschland mit Ausnahme Strohmeiers nicht auszumachen; nur Laien ${ }^{19}$ konstatieren gelegentlich „Unregierbarkeit", für die dann die Mehrheitswahl als Abhilfe gefordert wird. Dabei steht es nicht nur Wissenschaftlern gut an, Reformvorschläge an der Realität zu überprüfen und dabei auch den Blick über die Grenzen nicht zu scheuen.

18 Mit den Daten der Bundestagswahl 2005 käme dazu allerdings nur eine Koalition aus Union und Linkspartei in Frage, vgl. Harald Schoen, a.a.O., S. 863 f.

19 Vgl. Frank Decker, a.a.O., S. 861. 